\title{
Limiar epidêmico no Processo de Contato de uma Rede Complexa Multiescalar
}

\author{
Machado, A. P. ${ }^{1 *}$; Da Silva, S. L. ${ }^{2}$ \\ 1 Universidade Federal do Espírito Santo, Vitória, ES, Brasil. \\ 2 Instituto Federal do Espírito Santo, Vitória, ES, Brasil.
}

* e-mail: machadoap92@gmail.com.br

\begin{abstract}
Resumo
Investigamos um modelo de propagação de arboviroses urbanas transmitidas pelo Aedes aegypti. Através de um modelo computacional de processo de contato generalizado, constituído de uma rede multiescalar composta por uma rede unidimensional e uma rede livre de escalas, analisamos a influência que a mobilidade dos indivíduos de uma população acarreta na propagação da doença.
\end{abstract}

\begin{abstract}
We investigated an urban arbovirus spread model transmitted by Aedes aegypti. Through a computational model of generalized contact process, consists for a multiscale network composed of a one-dimension network and a scale free network, we analyzed the influence that the mobility of the individuals in a population cause in the spread of disease.
\end{abstract}

Keywords (Palavras chaves): redes complexas, propagação de doenças, limiar epidêmico.

\section{Introdução}

Em junho de 2009, a Organização Mundial de Saúde declarou pandemia para a gripe $A(\mathrm{H} 1 \mathrm{~N} 1)$ [1]. $\mathrm{O}$ espalhamento do vírus $\mathrm{H} 1 \mathrm{~N} 1$ ocorreu de forma rápida devido a fácil locomoção das pessoas. A doença teve sua evolução prevista antes de atingir o pico de espalhamento através da utilização da teoria de redes que, entre outras coisas, levou em consideração a aviação civil internacional [2] utilizando dados da International Air Transport Association (IATA) e Official Airline Guide (OAG) [3].

No Brasil, além de surtos de gripe comuns no inverno temos as arboviroses urbanas transmitidas pelo mosquito Aedes aegypti, que são a dengue, chikungunya e Zika. O boletim epidemiológico 02 da Secretaria de Vigilância em Saúde do Ministério da Saúde [4] relatou mais de 1,5 milhão de casos de dengue em 2019; chikungunya e Zika foram 132.205 e 10.768, respectivamente.

O ciclo de transmissão das arboviroses urbanas transmitidas pelo mosquito do gênero Aedes ocorre através da dinâmica homem-mosquito-homem. Neste trabalho, investigamos essa dinâmica através de simulação computacional de um modelo de Processo de Contato Generalizado (PCG), que utiliza uma rede complexa multiescalar no qual o contágio local entre os indivíduos ocorre pela rede regular linear unidimensional e uma rede livre de escalas representando a mobilidade das pessoas quando as mesmas se deslocam para o trabalho, por exemplo.

O PCG é um modelo simples, de rápida confecção e curta duração no tempo de processamento computacional e tem como objetivo reproduzir o cenário epidêmico auxiliando em estratégias de combate à doença.

\section{Ciência de redes}

Os sistemas complexos estão em toda a parte, estando presente no biológico, tecnológico e também no social. A sociedade requer a cooperação entre bilhões de indivíduos. A infraestrutura de telecomunicação requer a integração de bilhões de celulares com computadores e satélites. Nossa capacidade de raciocinar e compreender o mundo necessita da atividade de bilhões de neurônios em nosso cérebro [5].

A história da ciência de redes tem início no século XVIII com um enigma: as pontes de Königsberg. O enigma consistia em saber se era possível uma pessoa atravessar as sete pontes da cidade sem passar por elas duas vezes. 
Quem resolveu o problema foi o matemático suíço Leonhard Euler transformando a cidade e suas sete pontes em um grafo. Ele transformou as porções de terra em vértices e as pontes em conexões, conforme Figura 1.
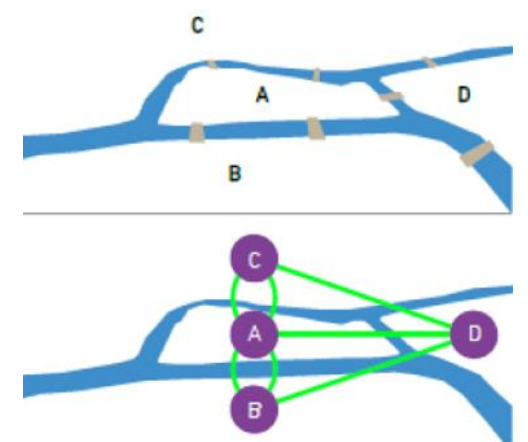

Figura 1: llustração esquemática dos quatro pedaços de terra e sete pontes de Königsberg. Euler construiu um grafo que tinha quatro nós ( $A$, B, C, D), correspondente a um pedaço de terra, e sete ligações, correspondendo as pontes. Fonte: [5]

Euler chegou à conclusão que era impossível uma pessoa atravessar as sete pontes da cidade sem passar por elas duas vezes, pois deveria haver apenas dois vértices com número de ligações ímpar, porém o desafio possui quatro $(A, B, C, D)$. Sendo assim, não existe uma forma de passar por todos os caminhos sem passar por ele novamente.

Grafos ou redes podem ser definidas como a relação entre os componentes do sistema, chamados de nó ou vértice, e as interações diretas entre eles, as ligações ou conexões [5].

As redes podem ser classificadas em duas classes: homogêneas e heterogêneas. Nas redes heterogêneas, no qual redes livres de escala se destacam, os nós não possuem o mesmo número médio de ligações (Figura $2 a$ e $2 b$ ). Já nas redes homogêneas, nas quais os grafos aleatórios e as redes regulares (euclidianas) são encontradas, cada nó possui aproximadamente 0 mesmo número de ligações (Figura 2c e 2d). (a)
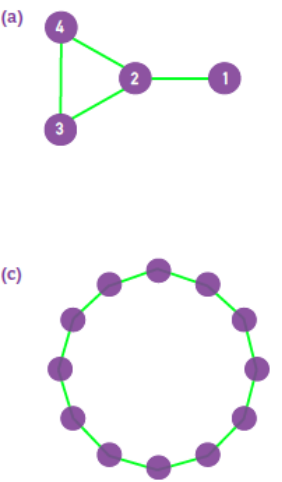

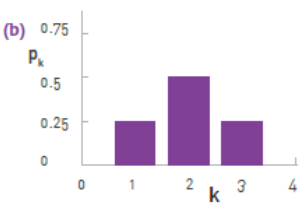

(d)

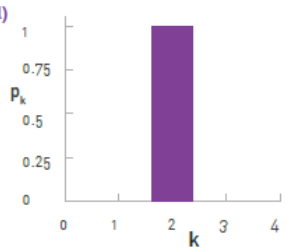

Figura 2: Em (a) e (b) temos uma rede com $\mathrm{N}=4$ nós com as seguintes funções de distribuição e grau: $\mathrm{p} 1=0.25$ e k1 $=1$, p2 $=0.5$ e k2 = 2, p3 $=0.25$ e $\mathrm{k} 3=2$. Já em (c) e (d) temos uma rede unidimensional em que cada nó tem o mesmo grau $\mathrm{k}=2$. Fonte: [5]
A Figura 2 ainda apresenta a variável $p_{k}$ que é a função de distribuição. Ela dá a probabilidade de um nó selecionado aleatoriamente ter exatamente $k_{i}$ conexões, no qual $k$ é chamado grau.

A função de distribuição de uma rede aleatória é uma distribuição de Poisson com um pico no número médio de ligações. Em redes livres de escala (SF) a função de distribuição possui uma lei de potência, assim

$$
\mathrm{p}_{\mathrm{k}} \approx \mathrm{k}^{-\gamma}
$$

Propriedades da SF depende do valor do expoente $\mathrm{Y}$. Sistemas em que $y \leq 2$ são chamados anômalos e 0 número de conexões ao nó mais conectado cresce mais rápido que o tamanho da rede, ou seja, para $N$ (número de nós) muito grande a função de distribuição $p_{k}$ vai exceder o número de nós, dessa forma, o valor médio de $k$ diverge quando $N$ tende ao infinito. Para $2 \leq y \leq 30$ regime é livre de escalas e o valor médio de $k$ converge quando $N$ tende ao infinito, porém o valor médio de $k^{2}$, não. Por fim, quando $y \geq 3$ tanto o valor médio de $k$ quanto de $k^{2}$ convergem e entram no regime de rede aleatória.

O modelo PCG considera interações entre vizinhos próximos (rede regular linear) e também interações de longo alcance (rede livre de escalas), portanto o modelo conta com uma rede complexa multiescalar.

\section{Metodologia}

O modelo PCG é uma generalização do modelo Processo de Contato (PC). O PC foi proposto em 1974 [6] por Harris como modelo simples de propagação de uma epidemia, considerando a interação local entre os indivíduos. Esse modelo foi um dos primeiros a apresentar um comportamento crítico não trivial. Esse modelo tem sido usado para descrever diversos sistemas dinâmicos [7].

Muitos trabalhos tem mostrado que o PC pertence a classe de universalidade da Percolação Direcionada (PD) [7]. Outras generalizações do processo de contato têm sido apresentadas nos últimos anos mostrando evidências que esses sistemas não são pertencentes a classe PD quando interações de longo alcance são implementadas [8 - 14].

A hipótese de universalidade, proposta por Kadanoff [15] reduz a grande variedade de fenômenos críticos a um pequeno número de classes de equivalência, as chamadas classes de universalidade, que dependem apenas de alguns parâmetros fundamentais. Portanto, a existência da universalidade nos permite fazer previsões a respeito do comportamento crítico de sistemas em equilíbrio e fora do equilíbrio, mesmo em casos onde os sistemas sejam difíceis de serem modelados. Por isso a importância de estudar generalizações do modelo PC. 


\subsection{Modelo}

O Processo de Contato Generalizado é construído em uma rede regular unidimensional de tamanho $L$ com condições de contorno periódicas, no qual o vértice representa um indivíduo. A interação de curto alcance acontece entre um indivíduo localmente imóvel em contato com apenas seus vizinhos próximos, o vizinho da direita e da esquerda. A interação de longo alcance variado ocorre entre conexões de indivíduos obedecendo uma rede livre de escala criada com base no modelo proposto por Barabási [16].

O modelo de Barabási é uma estruturação básica para geração de uma rede livre de escalas, estabelecido por Albert-László Barabási, que consiste em uma rede com $M_{0}$ vértices desconectados, inicialmente. A cada instante de tempo é adicionado um novo vértice fazendo $M$ ligações preferencialmente com os vértices mais conectados, ou seja, usando-se uma probabilidade de conexões $\left(\mathrm{k}_{\mathrm{i}}\right)=\mathrm{k}_{\mathrm{i}} / \sum_{\mathrm{j}} \mathrm{k}_{\mathrm{j}}$, que é o número de conexão que um determinado vértice possui dividido pelo número total de conexões existentes.

Tal probabilidade é um mecanismo probabilístico, que foi chamada de conexão preferencial [5]. Se, por exemplo, um novo vértice tem a opção de se conectar com um de grau dois ou um de grau quatro, a probabilidade de se conectar ao de grau quatro é duas vezes maior. A Figura 3 nos mostra a evolução de uma rede modelo Barabási.

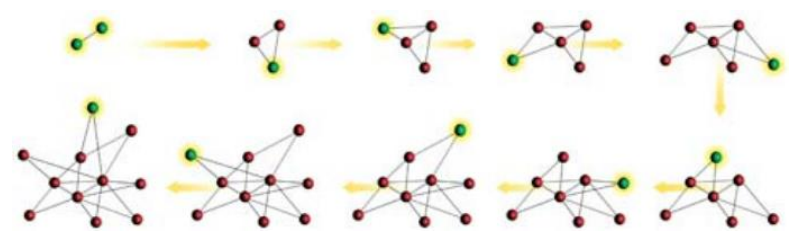

Figura 3: Evolução do Modelo Barabási. A sequência de imagens mostra nove passos subsequentes de um modelo Barabási. Circulos vazios marcam um novo vértice adicionado a rede. Fonte: [17].

Agora um indivíduo além do contato local com seus vizinhos próximos ele poderá interagir com indivíduos mais distantes, desde que eles estejam compartilhando uma conexão da rede livre de escala. Esse sistema é ilustrado na Figura 4.

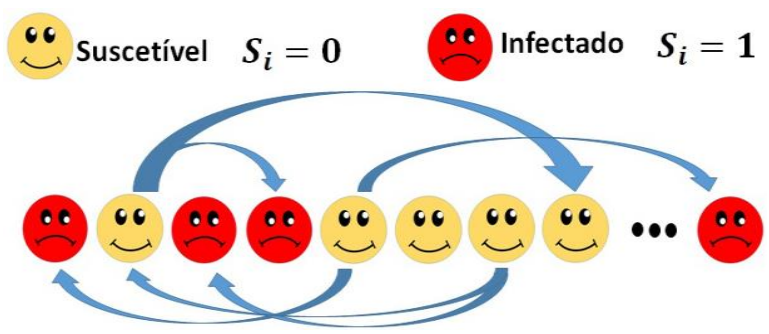

Figura 4: Rede multiescalar unidimensional com contatos locais (rede linear regular) e contatos globais (setas) através de uma rede livre de escala. Fonte: Autor.

O estado de um sistema é definido pela sequência $S_{1}$, $\mathrm{S}_{2}, \ldots, \mathrm{S} \mathrm{L}$ sendo que $\mathrm{S}_{\mathrm{i}}$ descreve o estado do sítio $i$ na rede $L$ unidimensional, onde $S_{i}=1$ significa indivíduo infectado ocupando o sítio $i$ e $S_{i}=0$ o indivíduo sadio. $\mathrm{Na}$ dinâmica de propagação aplicamos o modelo simplificado SIS (suscetível - infectado - suscetível) de propagação de doença. O indivíduo infectado, após se recuperar da doença, não se torna imune permanentemente, ou seja, ele pode contraí-la novamente.

A propagação da doença na rede regular é feita exclusivamente através do contato entre vizinhos próximos. Um indivíduo, escolhido aleatoriamente, poderá contrair a doença com uma probabilidade $p$ se pelo menos um dos seus vizinhos estiver infectado. Um indivíduo infectado poderá torna-se suscetível com uma probabilidade complementar $q=1-p$. A probabilidade de infecção $p$ e probabilidade de recuperação $q$ são definidos em função da taxa de infecção $\lambda$,

$$
\begin{aligned}
& \mathrm{p}=\frac{\lambda}{\lambda+1}, \\
& \mathrm{q}=\frac{1}{\lambda+1} .
\end{aligned}
$$

A transmissão da doença na rede livre de escala é feita por permuta de indivíduos que possuem conexão entre si. Para cada vértice infectado da rede livre de escala escolhe-se um outro vértice ao acaso com uma probabilidade dada por

$$
r=\frac{\alpha}{\alpha+1}
$$

em que a é a taxa de mobilidade.

Em seguida verifica-se a existência da conexão entre eles. Em caso afirmativo a permutação ocorrerá com uma probabilidade $\mathrm{p}_{\mathrm{k}}=N_{\mathrm{k}}(\mathrm{i}) / \mathrm{K}_{\text {total }} \mathrm{p}_{\mathrm{k}}=\mathrm{N}_{\mathrm{k}}(\mathrm{i}) / \mathrm{K}_{\text {total }}$, que dependerá da conectividade do vértice sorteado, ou seja, o número de conexões que este vértice $N_{k}(i) \mathrm{N}_{\mathrm{k}}(\mathrm{i})$ possui dividido pelo número total de conexões $K_{\text {total }} K_{\text {total }}$ da rede. Neste momento, um par de vértices (doentes ou saudáveis) da rede regular são escolhidos ao acaso e trocarão de posição. Esse mecanismo é responsável por difundir a doença através de interações de alcance variado. Terminado a propagação da doença nas duas redes um passo de tempo de Monte Carlo é contado.

Definido o tamanho $L$ da rede multiescalar, o estado inicial da evolução da epidemia foi estabelecido colocando apenas um indivíduo infectado (semente ativa) no centro da rede regular unidimensional. Em nossa simulação consideramos um sistema de tamanho $\mathrm{L}=500$ para análise temporal.

O modelo foi transcrito em linguagem de programação Fortran 90 e então compilado gerando os resultados discutidos na seção seguinte. 


\section{Discussão dos resultados}

O sistema é inicializado com uma semente ativa no centro da rede de tamanho $L=500$ e taxa de mobilidade $\alpha=2,0$. Temos nas Figura 5 (a) e (b) a representação da densidade de infectados $\rho$ pelo tempo. Na Figura 5 (a) para $\lambda=1,65$, $\rho$ atinge o regime absorvente após um determinado período de tempo, enquanto na Figura 5 (b) com $\lambda=1,90, \rho$ persiste na rede atingindo o estado estacionário. Esse cenário indica a existência de uma transição de fase no modelo, caracterizada por uma taxa de infecção crítica $\lambda_{c}$.
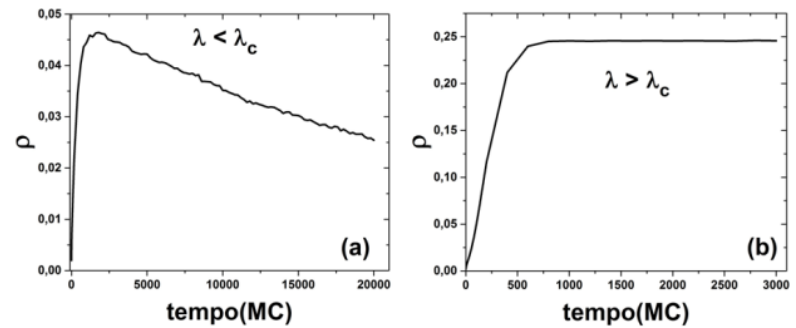

Figura 5: Densidade de infectados $\rho$ no modelo PCG com $L=500$ e $\alpha=$ 2,0. (a) Estado absorvente para $\lambda=1,65$ e em (b) estado estacionário para $\lambda=1,90$.
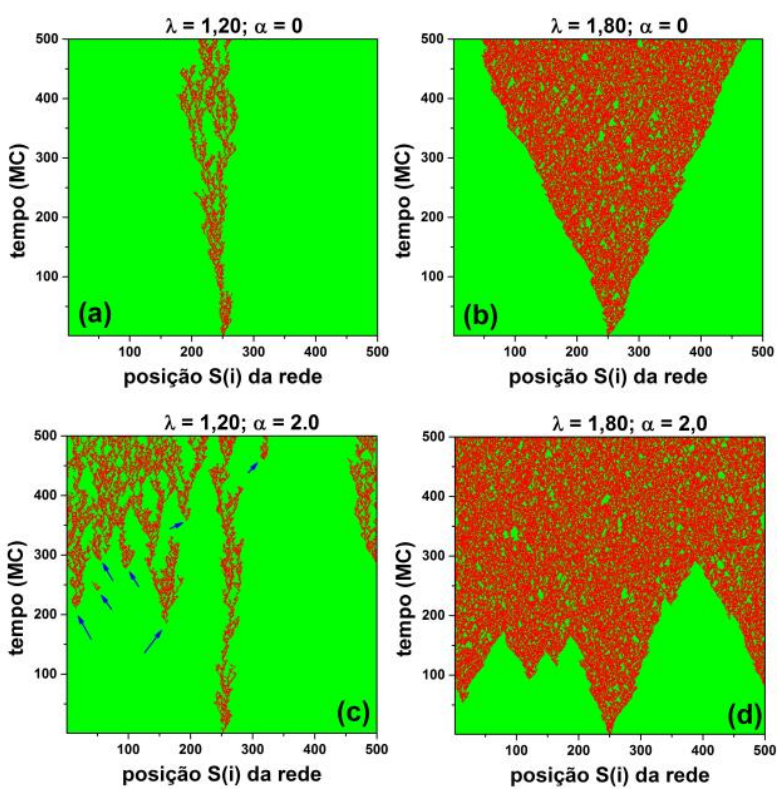

Figura 6: Evolução temporal para $L=500$ no modelo $P C(\alpha=0)$ para valores com (a) $\lambda<\lambda c$ e (b) $\lambda>\lambda c$. Em (c) $\lambda<\lambda c$ e (d) $\lambda>\lambda c$ estão o resultado para o modelo PCG com $\alpha=2.0$.

Além da taxa de infecção $\lambda$, o número de infectados em função do tempo depende também da taxa de mobilidade $\alpha$. A taxa de mobilidade exerce o papel de espalhar, não uniformemente, os infectados na rede, rompendo grandes e pequenas distâncias entre dois indivíduos da rede. Na Figura 6 apresentamos uma comparação quando se impõem no modelo uma taxa de mobilidade nula (PC) e uma taxa de mobilidade diferente de zero (PCG) na propagação temporal da doença. Novamente para $\lambda<\lambda_{c}, \rho$ atinge o regime absorvente Figura 6 (a) e (c)] e para $\lambda>\lambda_{c}$, $\rho$ alcança o regime estacionário Figura 6 (b) e (d)].
Os indivíduos mais distantes do centro da rede precisam de um tempo maior para ter contato com indivíduos infectados para $\alpha=0$. Utilizando os mesmos valores da taxa de infecção no modelo PCG, onde nossos resultados mostram que a permuta de infectados, que possuem uma conexão na rede livre de escala, favorece o surgimento de mais sementes ativas em outras regiões da rede ao longo do tempo [veja as setas na Figura 6 (c)]. Na Figura 6 (d) podemos observar que a distribuição de infectados consegue atingir regularmente todas as regiões da rede em um tempo menor do que é observado para o PC na Figura 6 (c). No PC os indivíduos da periferia da rede nunca seriam atingidos pela doença se $\lambda<\lambda_{c}$ ou levaria um tempo longo para algum deles terem contato, já que o único mecanismo de propagação é por vizinhos próximos. Por outro lado, no modelo PCG existe a possibilidade de surgir novas sementes ativas na periferia da rede, o que diminui o tempo para esses indivíduos terem contato com a doença.

\section{Conclusão}

O modelo PCG indica que a taxa de mobilidade $\alpha$ favorece o alcance variado, gerado por conexões livre de escala. A mobilidade de indivíduos via rede livre de escala proporciona uma distribuição mais uniforme dos indivíduos infectados na rede, aumentando a chance de atingir muitos indivíduos ao mesmo tempo. O estudo mais aprofundado da taxa de infecção crítica para diferentes valores de tamanho de rede e taxa de mobilidade podem responder se a dinâmica de propagação dessa doença poderá atingir o estado absorvente ou o estado estacionário.

\section{Referências}

[1] Organização Mundial de Saúde declara pandemia da nova gripe, São Paulo. Disponível em <http://g1.globo.com/Sites/Especiais/Noticias/0,,MUL11 91163-16726,00ORGANIZACAO+MUNDIAL+DE+SAUDE+DECLARA+ PANDEMIA+DA+NOVA+GRIPE.html>. Acesso em: 28 set. 2020.

[2] Ferreira, R. S. Processos dinâmicos com estados absorventes em redes complexas. Tese (doutorado em Física), Programa de Pós-Graduação em Física, Universidade Federal de Viçosa, Viçosa, 2013.

[3] Balcan, D. et al. BMC medicine, v. 7, p. 45, 2009.

[4] Boletim Epidemiológico 02, Brasília. Disponível em <http://portalarquivos2.saude.gov.br/images/pdf/2020/ja neiro/20/Boletim-epidemiologico-SVS-02-1-.pdf>.

Acesso em: 28 set. 2020.

[5] BARABÁSI, A. L. Network Science. [S.I.]: Cambridge University Press, 2016.

[6] HARRIS, T. E. The Annals of Probability, p. 969-988, 1974. 
[7] MARRO, J.; DICKMAN, R. Nonequilibrium phase transitions in lattice models. [S.I.]: Cambridge University Press, 2005.

[8] SILVA, M. da et al. Physica A: Statistical Mechanics and its Applications, v. 404, p. 271-278, 2014.

[9] SILVA, S.; FERREIRA, J.; MARTINS, M. Elsevier, v. 377, n. 2, p. 689-697, 2007.

[10] FERREIRA, S. C.; MARTINS, M. L. Physical Review E, APS, v. 76, n. 3, p. 036112, 2007.

[11] PASTOR-SATORRAS, R.; VESPIGNANI, A. Physical review letters, APS, v. 86, n. 14, p. 3200, 2001.

[12] CASTELLANO, C.; PASTOR-SATORRAS, R. Physical review letters, APS, v. 100, n. 14, p. 148701 , 2008.
[13] FERREIRA, S. C. et al. Physical Review E, APS, v. 84, n. 6, p. 066102, 2011.

[14] NOH, J. D.; PARK, H. Physical Review E, APS, v. 79, n. 5, p. 056115, 2009.

[15] HINIRCHSEN, H.; LUBECK, S. Non-Equilibrium Phase Transitions, Volume I: Absorbing Phase Transitions. Theoretical and Mathematical Physics. [S.I.]: Springer, 2008.

[16] Barabási, A. L.; Albert, R.; \& Jeong, H. Phys. A, v.272, p. 173-187, 1999.

[17] Barabási, Albert-László, and Eric Bonabeau. Scientific american 288.5 (2003): 60-69. 